\title{
Exploring allele specific methylation in drug dependence susceptibility
}

\author{
${\text { Laura Pineda-Cirera }{ }^{\text {a,b,c,d }} \text {, Judit Cabana-Domínguez }}_{\text {a,b,c,d }}^{\text {, Lara Grau-López }}{ }^{\text {e,f,g,h }}$, \\ Constanza Daigre $^{\text {e,f,g,h }}$, Cristina Sánchez-Mora ${ }^{\text {a,e,g,i }}$, Raul Felipe Palma-Álvarez ${ }^{\text {ef, g, h, }}$ \\ Josep Antoni Ramos-Quiroga e, f, g, h, Marta Ribasés ${ }^{\text {a,e, , , , }}$, Bru Cormand ${ }^{\text {a,b, c, d, 1, ** , }}$

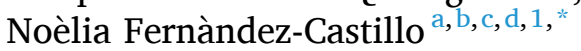

\footnotetext{
${ }^{a}$ Departament de Genètica, Microbiologia i Estadística, Facultat de Biologia, Universitat de Barcelona, Catalonia, Spain

${ }^{\mathrm{b}}$ Institut de Biomedicina de la Universitat de Barcelona (IBUB), Catalonia, Spain

${ }^{\mathrm{c}}$ Centro de Investigación Biomédica en Red de Enfermedades Raras (CIBERER), Spain

' Institut de Recerca Sant Joan de Déu (IR-SJD), Esplugues de Llobregat, Catalonia, Spain

e Department of Psychiatry, Hospital Universitari Vall d'Hebron, Vall d'Hebron Research Institute (VHIR), Universitat Autònoma de Barcelona, Barcelona, Catalonia,

Spain

${ }^{\mathrm{f}}$ Group of Psychiatry, Mental Health and Addictions, Catalonia, Spain

${ }^{\mathrm{g}}$ Biomedical Network Research Centre on Mental Health (CIBERSAM), Instituto de Salud Carlos III, Madrid, Spain

${ }^{\mathrm{h}}$ Department of Psychiatry and Legal Medicine, Universitat Autònoma de Barcelona, Barcelona, Catalonia, Spain

i Psychiatric Genetics Unit, Group of Psychiatry, Mental Health and Addiction, Vall d'Hebron Research Institute (VHIR), Universitat Autonoma de Barcelona, Barcelona, Spain
}

\section{A R T I C L E I N F O}

\section{Keywords:}

CTNNBL1

SCP2

ECHDC2

Brain DNA methylation

SNP

Association

\begin{abstract}
A B S T R A T
Drug dependence is a neuropsychiatric condition that involves genetic, epigenetic and environmental factors. Allele-specific methylation (ASM) is a common and stable epigenetic mechanism that involves genetic variants correlating with differential levels of methylation at CpG sites. We selected 182 single-nucleotide polymorphisms (SNPs) described to influence cis ASM in human brain regions to evaluate their possible contribution to drug dependence susceptibility. We performed a case-control association study in a discovery sample of 578 drugdependent patients (including 428 cocaine-dependent subjects) and 656 controls from Spain, and then, we followed-up the significant associations in an independent sample of 1119 cases (including 589 cocainedependent subjects) and 1092 controls. In the discovery sample, we identified five nominal associations, one of them replicated in the follow-up sample (rs6020251). The pooled analysis revealed an association between drug dependence and rs6020251 but also rs11585570, both overcoming the Bonferroni correction for multiple testing. We performed the same analysis considering only cocaine-dependent patients and obtained similar results. The rs6020251 variant correlates with differential methylation levels of cg17974185 and lies in the first intron of the CTNNBL1 gene, in a genomic region with multiple histone marks related to enhancer and promoter regions in brain. Rs11585570 is an eQTL in brain and blood for the SCP2 and ECHDC2 genes and correlates with differential methylation of cg27535305 and cg13461509, located in the promoter regions of both genes. To conclude, using an approach that combines genetic and epigenetic data, we highlighted the CTNNBL1, SCP2 and ECHDC2 genes as potential contributors to drug dependence susceptibility.
\end{abstract}

\section{Introduction}

Drug use is a major health problem worldwide, with about 35 million people, or $0.7 \%$ of the global adult population being affected by illegal substance use disorders (UNODC, 2019) and 5.1\% affected by alcohol use disorders (World Health Organization, 2018). Drug dependence is a

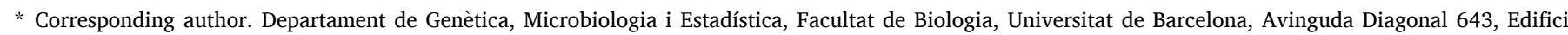
Prevosti, $1^{\text {a }}$ Planta, Sala 134, 08028, Barcelona, Catalonia, Spain.

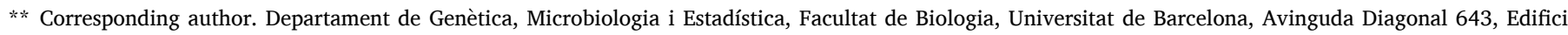
Prevosti, $3^{\text {a }}$ planta, 08028, Barcelona, Catalonia, Spain.

E-mail addresses: bcormand@ub.edu (B. Cormand), noefernandez@ub.edu (N. Fernàndez-Castillo).

${ }^{1}$ Senior authors that equally contributed.
} 
complex psychiatric disorder in which genetic and environmental risk factors are involved. Heritability estimates of addiction range from 39 to $72 \%$, depending on the drug of abuse (Ducci and Goldman, 2012). Although multiple studies have been performed to understand the genetic basis of drug dependence (reviewed by Bühler et al., 2015; Edenberg et al., 2019; Jensen, 2016; Mulligan, 2019), the underlying molecular mechanisms are still largely unknown. It has been widely suggested that epigenetics, and particularly DNA methylation, may have a role in addiction (Cadet et al., 2016; Hamilton and Nestler, 2019), as the interplay between genetic and environmental factors. Furthermore, DNA methylation is implicated in the regulation of multiple brain events like neurogenesis, differentiation and brain development (Ladd-Acosta et al., 2007) in addition to synaptic plasticity, memory formation and acquired behavior (Bashtrykov and Jeltsch, 2017), many of them involved in the transition from drug use to dependence.

DNA methylation is a common and stable epigenetic mechanism catalyzed by DNA methyltransferases (DNMT) that add a methyl group usually to the cytosine of a CpG dinucleotide (Klose and Bird, 2006). DNMT expression alterations have been linked to drug dependence and to other psychiatric disorders like schizophrenia (Ajonijebu et al., 2017). Regarding specific drugs, methamphetamine and cocaine administration have been related to expression changes of some DNMT isoforms that lead to changes in DNA methylation in the brain (Cadet et al., 2016). Furthermore, cocaine has also been related to an increased expression of methyl-CpG binding protein 2 (Cadet et al., 2016). Based on growing evidence that links DNA methylation with addiction, a pretreatment with methionine, a methyl donor, has been proposed as a possible therapy for this disorder (Brown and Feng, 2017).

Allele-specific methylation (ASM) is an epigenetic mechanism in which the genotype of a genetic variation correlates with DNA methylation (usually of a CpG dinucleotide) either in cis or in trans (Meaburn et al., 2010). It is quantitative and heterogeneous across tissues and individuals (Meaburn et al., 2010) and can lead to allele-specific expression (ASE) through the alteration of the activity of gene promoters (Gaur et al., 2013). During the last decade, several studies have focused on the identification of ASM SNPs in multiple human tissues (Do et al., 2017), some of them in brain regions (Gibbs et al., 2010; Zhang et al., 2010). Remarkably, ASM has previously been associated with psychiatric disorders, such as autism (Andrews et al., 2017), bipolar disorder (Chuang et al., 2013), schizophrenia (Gagliano et al., 2016; Wu and Pan, 2019) or ADHD (Pineda-Cirera et al., 2019).

The present study aims at elucidating the contribution of ASM to drug dependence susceptibility through a case-control study in a Spanish sample with European ancestry.

\section{Material and methods}

\subsection{Subjects}

The discovery sample consisted of 578 substance-dependent patients (including 428 cocaine-dependent subjects) and 656 control individuals and the follow-up sample included 1119 substance-dependent patients (with 843 cocaine-dependent subjects) and 1092 control individuals (Fig. 1 and Supplementary Table 1). Both samples had the same sex ratio in the case and control groups and all individuals were Spanish, with European ancestry and unrelated. Patients were diagnosed under DSMIV-TR criteria (Diagnostic and Statistical Manual of Mental Disorders, 4th ed., text revision) and the SCID-I (Structured Clinical Interview) (First et al., 1997) at the Addiction and Dual Diagnosis Section of the Department of Psychiatry at Hospital Universitari Vall d'Hebron (Barcelona, Spain). Patients were included in the study if they met the criteria for dependence to at least one drug of abuse (Fig. 1). The control individuals were blood donors recruited at the Blood and Tissues Bank of Barcelona, who had not injected drugs intravenously. The study was approved by the ethics committee of our institution according to the Helsinki Declaration, the study design was reviewed by an appropriate
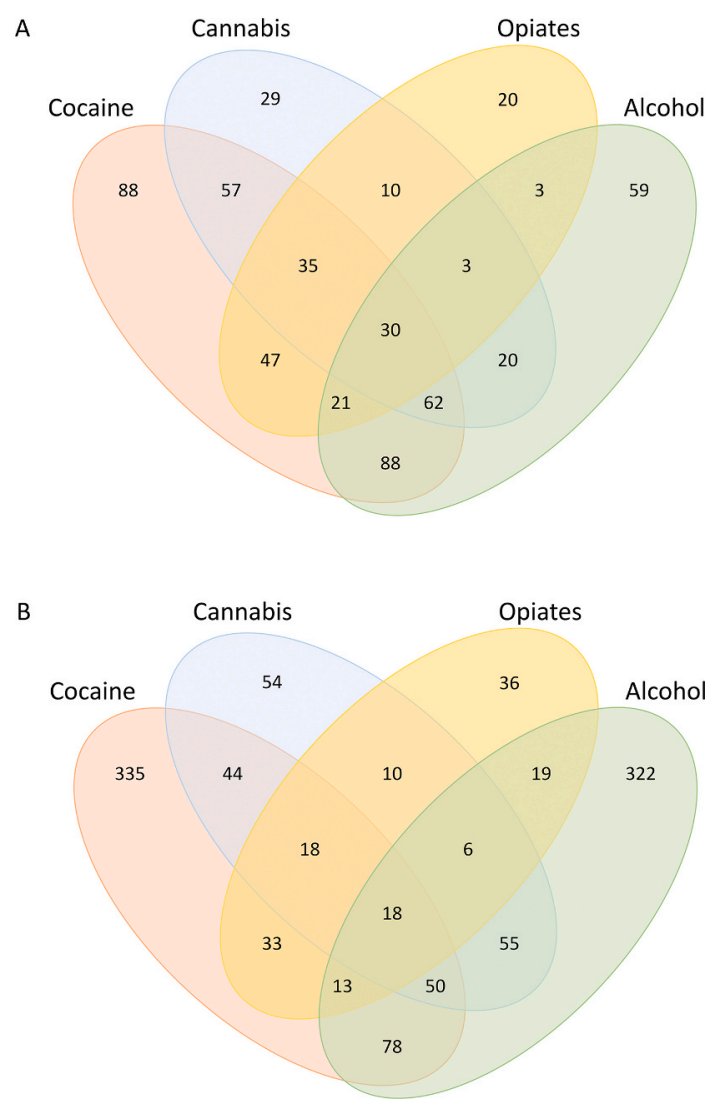

Fig. 1. Distribution of the four main addictions in the patients' sample (cocaine, cannabis, alcohol and opiates). Other addictions with a frequency lower than $10 \%$ are not displayed. A) Discovery sample. B) Replication sample. The percentage of cases addicted to more than one drug is $65.7 \%$ (A) and $31.5 \%$ (B).

ethical committee and informed consent was obtained from all participants. Population stratification was previously discarded in part of our sample (Fernàndez-Castillo et al., 2013).

\subsection{DNA isolation and quantification}

Genomic DNA was isolated from peripheral blood using the saltingout method (Miller et al., 1988). DNA concentration of all samples was measured on a NanoDrop ND-1000 spectrophotometer (NanoDrop Technologies, Thermo Fisher Scientific Inc., Wilmington, DE, USA). DNA concentration for the discovery sample was also quantified using Picogreen (Life Technologies, Carlsbad, CA, USA) with a Glomax ${ }^{\circledR}-M u l t i$ Detection System (Promega, Madison, WI, USA).

\subsection{Selection of allele-specific methylation SNPs and genotyping}

The SNP selection was made based on two previous studies that listed ASM variants for multiple brain regions of postmortem human samples (Gibbs et al., 2010; Zhang et al., 2010). Gibbs et al. (2010) considered four brain regions (cerebellum, frontal cortex, caudal pons and temporal cortex) of 150 subjects and Zhang et al. (2010) used only the cerebellum of another group of 153 subjects. Genomic variants located within the probe sequences used in methylation arrays may affect their binding affinity and cause inaccurate methylation measurements. To avoid potential artefacts in our results, we confirmed that the probes used to detect the CpG sites reported by us do not target genomic regions containing common genetic variants.

In the study by Zhang et al. (2010) a total of 12,117 SNP-CpG pairs associations were reported in cerebellum and Gibbs et al. (2010) listed a 
total of 12,135 SNP-CpG pairs in frontal cortex, 11,374 in caudal pons, 16,734 in temporal cortex and 12,102 in cerebellum (Fig. 2). We combined the two studies and obtained a total of 43,132 SNP-CpG pairs involving 33,944 different SNPs and 5306 CpG sites (Fig. 2).

We selected ASM SNPs showing high correlations between the SNP and the methylation levels of a CpG site in cis (threshold set at $\mathrm{R}^{2} \geq 0.5$ ). Per each CpG site, we selected the SNP showing the highest $R^{2}$ and, in case of a tie, the SNP closest to the CpG site (Fig. 2). In almost all cases the SNP showing the highest $\mathrm{R}^{2}$ matched across tissues. However, occasionally this did not occur, so that for one of the tissues a variant different from the selected SNP displayed a higher correlation $\left(\mathrm{R}^{2}\right)$. In those cases, we calculated the linkage disequilibrium (LD) between the selected variant and the variant of highest $\mathrm{R}^{2}$ in the particular tissue to take into account information redundancy. Thus, if the two SNPs were in high LD ( $\mathrm{r}^{2} \geq 0.75$ ), we did not consider the second SNP, but in case of lower LD values $\left(\mathrm{r}^{2}<0.75\right)$, we also selected the SNP with higher $\mathrm{R}^{2}$ for this particular tissue. Using these criteria, we ended up with 182 SNPs (Fig. 2 and Supplementary Table 2).

The linkage disequilibrium (LD) analysis was carried out using Haploview software (Barrett, 2009) with the Central European (CEU) reference panel from HapMap (www.hapmap.org; release 23).

The genotyping of the discovery sample was performed using the BioMark HD System (Fluidigm, CA, USA) by the "Servicio General de Genómica: Unidad de Secuenciación y Genotipado de la Universidad del País Vasco", Spain. The genotyping of the replication sample was done with the KASP technology (LGC Biosearch Technologies, CA, USA) and was performed by LGC genomics, UK. Both assays are based on competitive allele-specific PCR that enable bi-allelic scoring of single nucleotide polymorphisms (SNPs) through a fluorescence-based detection. Primers used under the two genotyping technologies were designed by the corresponding company. Duplicates of some samples and negative controls were included in the genotyping of both the discovery and replication samples.

\subsection{Statistical analysis}

First, we assessed if there were significant age differences between cases and controls since means differed considerably (Supplementary
Table 1). The Saphiro-Wilk test determined the data was not fitting a normal distribution and hence, we used the non-parametric test U MannWhitney with SPSS22 (SPSS Inc., Chicago, IL, USA) and obtained significant differences in age between cases and controls both in the discovery and in the replication samples.

We excluded from the association study those SNPs with more than $5 \%$ missing genotypes. Hardy-Weinberg equilibrium (HWE) was tested for all the SNPs separately in controls and cases using the SNPassoc package of the R library (González et al., 2007), and those showing departure from HWE $(\mathrm{p}<0.01)$ were excluded. The case-control association test was carried out using the same package, which considers four different models: additive, dominant, recessive and codominant. As we detected differences in age between cases and controls, we also calculated and presented all the p-values adjusted by age.

For the discovery sample we only considered the additive model to limit the number of tests. The SNPs that showed nominal associations in the discovery sample, and remained significant after adjusting by age, were subsequently assessed in the replica sample under the different genetic models. Then, we conducted a pooled analysis of all the individuals from the discovery and replica samples under the model showing the best performance. Finally, we assessed whether the associations obtained survived the Bonferroni correction for multiple testing by setting the p-value thresholds for each analysis (discovery sample: $\mathrm{p}$ $=2.9 \mathrm{E}-04(0.05 / 173$ tests; considering 173 SNPs and one genetic model); replication sample: $\mathrm{p}=2.5 \mathrm{E}-03$ (0.05/20 tests; considering 5 SNPs and four models); and pooled analysis: $p=1 \mathrm{E}-02$ (0.05/5 tests; considering 5 SNPs and one model)). We considered that one SNP survived the Bonferroni correction for multiple testing if both p-values, adjusted and non-adjusted by age, were under the threshold.

\subsection{Functional annotation}

We investigated the possible functional role of the associated ASM SNPs using different tools. First, we evaluated the predicted effect of the variant on the binding of transcription factors using the FeatSNP tool (Ma et al., 2019). Second, we evaluated whether the ASM SNPs are located in a region with histone marks related to enhancer regions (H3K4me1 and H3K27ac) and promoter regions (H3K4me3 and

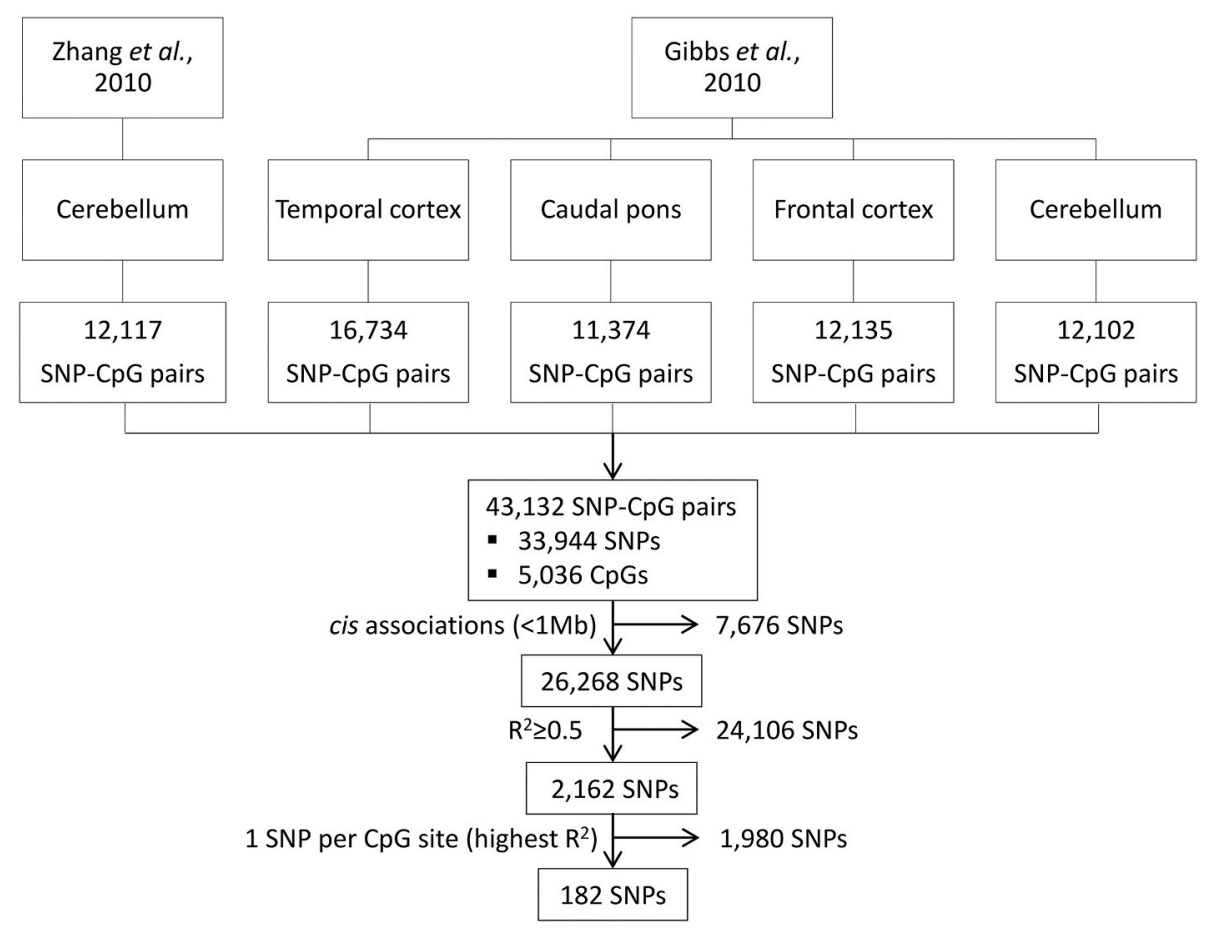

Fig. 2. Selection of allele-specific methylation (ASM) SNPs from two previous studies. 
H3K9ac) using the Haploreg v4.1 tool (Ward and Kellis, 2016) and considering all the available brain regions. Finally, we inspected the possible effect of the associated variants on gene expression checking if they were described to be eQTLs (expression quantitative trait loci) using GTEx data (Release V8) (Lonsdale et al., 2013). We considered eQTL information for blood and from all available brain tissues.

\section{Results}

The aim of the present study was to assess the contribution to drug dependence susceptibility of SNPs showing allele-specific methylation in brain regions. Starting from two previous studies (Gibbs et al., 2010; Zhang et al., 2010) that report more than 43,000 SNP-CpG pairs in several brain tissues, we selected 182 SNPs (Fig. 2 and Supplementary Table 2). The selection was restricted to variants showing a high correlation in cis with methylation levels $\left(\mathrm{R}^{2} \geq 0.5\right)$, taking only one SNP per $\mathrm{CpG}$ site to avoid redundancies. We further excluded $8 \mathrm{SNPs}$ from the analysis that showed more than $5 \%$ of missing genotypes and one SNP that deviated from Hardy-Weinberg equilibrium (Supplementary Table 2). Finally, a total of 173 SNPs were investigated through a case-control association study in a Spanish sample of drug-dependent individuals and the corresponding controls, all with European ancestry (Supplementary Table 2).

In the discovery sample (578 cases and 656 controls) we tested the additive model and identified five SNPs nominally associated with drug dependence: rs823080, rs3766612, rs6020251, rs9891018 and rs11585570 (Supplementary Table 3). We followed up these five associations in an independent sample (1119 cases and 1092 controls) considering the additive, dominant, recessive and codominant models and the association between rs6020251 and drug dependence was replicated under the recessive one $\left(\mathrm{P}=4.79 \mathrm{E}-02\right.$; Padj $\left._{\text {age }}=3.86 \mathrm{E}-02\right)$ (Table 1A and Supplementary Table 4). We also performed a pooled analysis under the recessive model including the discovery and replication samples where rs6020251 ( $\mathrm{P}=3.70 \mathrm{E}-03$ Padj $\left._{\mathrm{age}}=2.35 \mathrm{E}-03\right)$ and also rs11585570 ( $\left.\mathrm{P}=1.54 \mathrm{E}-03 ; \mathrm{Padj}_{\mathrm{age}}=7.65 \mathrm{E}-03\right)$ remained associated with drug dependence, overcoming the Bonferroni correction for multiple testing correction (Table 1A).

We then performed the association analysis for the subset of cocainedependent patients (about $60 \%$ of our patients' sample) under the recessive model. Both variants showed the same direction of association as in the analysis for drug dependence, and interestingly, rs6020251 survived the Bonferroni correction for multiple testing in the pooled analysis (Table 1B).
Rs6020251 correlated with differential levels of methylation of $\operatorname{cg} 17974185\left(R^{2} \geq 0.6\right)$ in all four brain areas studied (Gibbs et al., 2010; Zhang et al., 2010), with the risk allele A associated with higher levels of methylation (Fig. 3A and Table 2). The SNP is located 26 bp upstream from the CpG site (cg17974185) and both lie within the first intron of the CTNNBL1 reference transcript isoform, about 900 bp downstream from the transcription start site (TSS) and at the $5^{\prime}$ end of other isoforms of the same gene (49-82 kb 5' from the TSS of the other isoforms) (Fig. 3A). Remarkably, this variant lies in a region with multiple brain histone marks related to enhancer and promoter regions (Table 2 and Supplementary Table 5). Finally, it is also predicted to increase the binding affinity of four transcription factors: NKX3-2, NKX3-1, ZNF354C and ISL2 (Table 2 and Supplementary Table 6). It is important to highlight that the SNP rs6020251 is in high LD $\left(\mathrm{r}^{2} \geq 0.85\right)$ with other SNPs that also correlated with differences in methylation of cg17974185 and were not genotyped in this study (Supplementary Table 7).

On the other hand, rs11585570 correlates with differential levels of methylation of cg27535305 $\left(\mathrm{R}^{2}>0.5\right)$ in three brain areas, but also with $\operatorname{cg} 13461509\left(R^{2} \geq 0.27\right)$ in three regions (Fig. 3B and Table 2). In both cases, the risk allele $\mathrm{C}$ is associated with higher levels of methylation (Fig. 3B and Table 2). This variant is an eQTL for SCP2 and ECHDC2 in different brain areas and in blood, with the risk allele $\mathrm{C}$ associated with less gene expression (Fig. 3B and Table 2). Interestingly, both CpG sites, cg27535305 and cg13461509, lie in the possible promoter regions of the SCP2 and ECHDC2 genes (Fig. 3B). The cg27535305 lies about 400 bp and $5.3 \mathrm{~kb}$ upstream from the TSS of the SCP2 and the ECHDC2 reference transcripts, respectively. The cg13461509 lies about $5.4 \mathrm{~kb}$ and $200 \mathrm{bp}$ upstream from the TSS of the SCP2 and the ECHDC2 reference transcripts, respectively. Furthermore, rs 11585570 is predicted to affect the binding of the transcription factor HOXA5, with an increase in the affinity when the risk allele $C$ is present (Table 2 and Supplementary Table 6). The SNP rs11585570 is also in high LD with other SNPs affecting methylation of these two CpG sites and with a possible functional effect (Supplementary Table 7).

\section{Discussion}

Epigenetic factors are known to mediate the link between genetics and environment, which makes them ideal candidates as risk factors for psychiatric conditions. DNA methylation, and particularly ASM, has been investigated as a potential etiological factor in multiple psychiatric disorders. An enrichment of ASM SNPs in genome-wide association studies (GWAS) has been reported in psychiatric disorders like

Table 1

Significant associations under the recessive model with A) drug dependence and B) cocaine dependence.

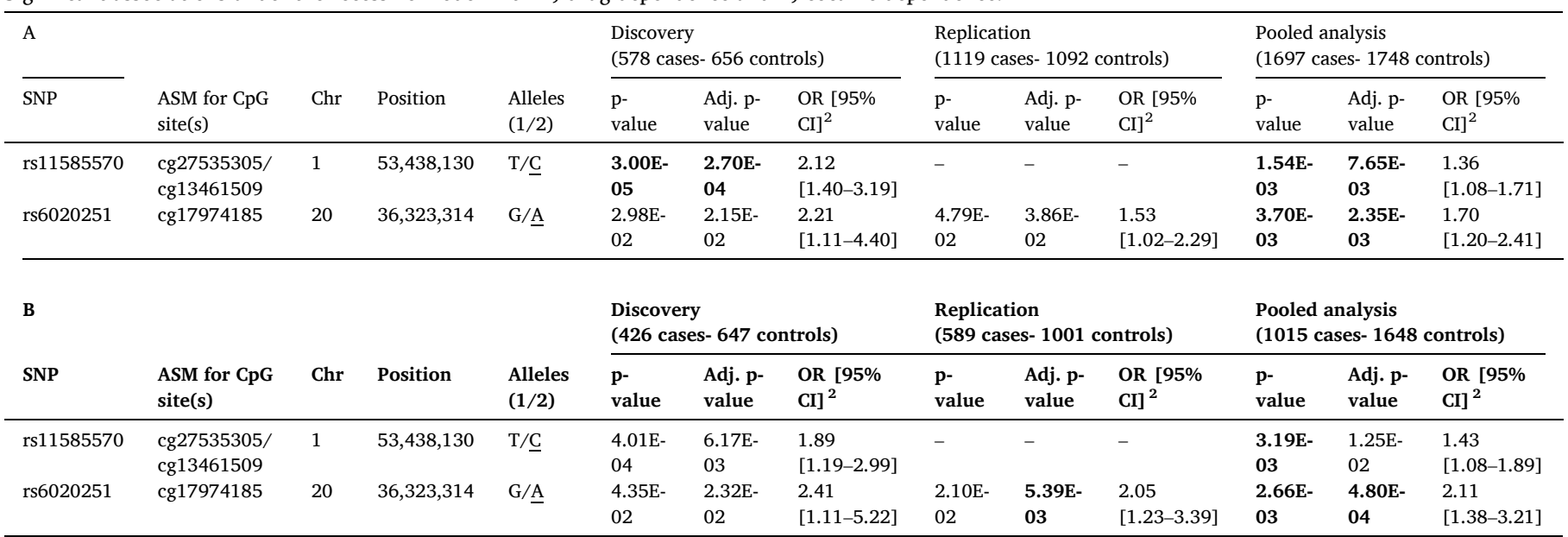

SNP: Single Nucleotide Polymorphism; ASM: Allele-Specific Methylation; Chr: Chromosome; Position: build hg19; Adj. P-value: p-value adjusted by age; All alleles are reported in the forward strand; Risk allele underlined; In bold: associations overcoming the Bonferroni correction for multiple testing (discovery sample: $\mathrm{p}<2.9 \mathrm{E}-04$; replication sample: $\mathrm{p}<2.5 \mathrm{E}-03$; pooled analysis: $\mathrm{p}<1 \mathrm{E}-02$ ). 


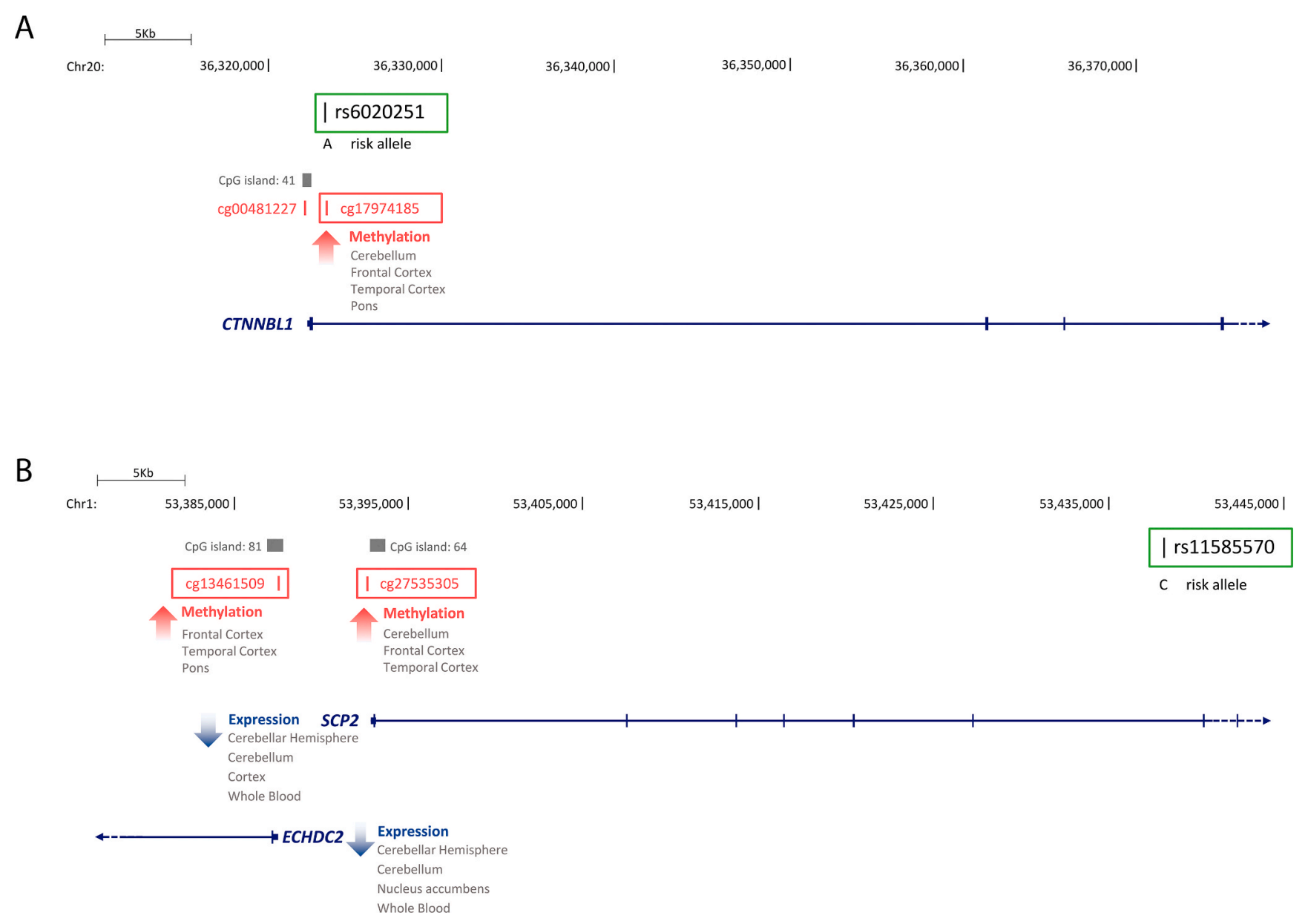

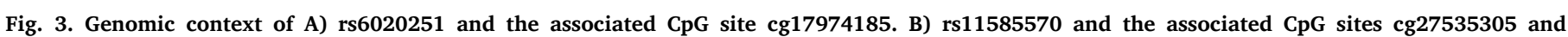

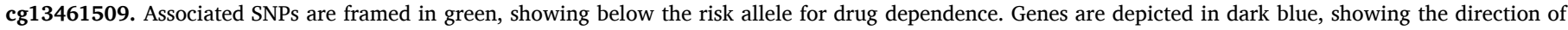

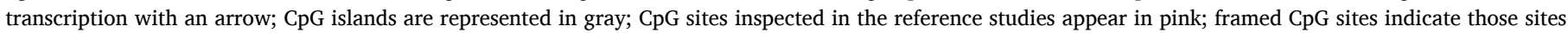

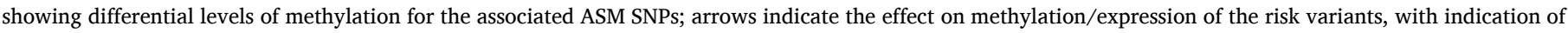

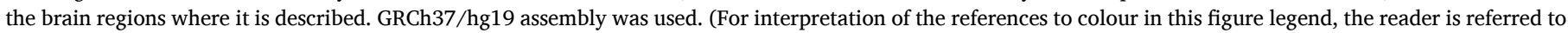
the Web version of this article.)

schizophrenia and ADHD (Gagliano et al., 2016; Pineda-Cirera et al., 2019). In the present study, we have explored the possible contribution to substance use disorders of ASM variants previously described in the human brain and studied their possible impact on gene expression. Thus, we have been able to identify two genetic variants (rs6020251 and rs11585570), that influence methylation at nearby CpG sites, associated with drug dependence and particularly with cocaine dependence through a case-control association study in a Spanish sample with European ancestry.

In general, ASM is associated with genetic variation in cis (up to 1 $\mathrm{Mb}$ ), although there is also a proportion of variation in trans. Thus, SNPs located far away from $\mathrm{CpG}$ sites can also have an impact on methylation (Meaburn et al., 2010). There are multiple mechanisms that can lead to ASM, for instance SNPs that affect the binding of regulatory proteins like transcription factors or the transcriptional repressor CTCF (Do et al., 2016; Tycko, 2010). Any alteration of these protein-DNA interactions by the effect of SNP variants can lead to changes in the 3D structure of the DNA and therefore, produce effects on distant CpG sites. In the two studies that we used to select ASM in brain, most of the reported effects were in cis (up to $1 \mathrm{Mb}$ ). Thus, Gibbs calculated an average distance of $81 \mathrm{~kb}$ between the SNP and the CpG site in associations in cis, which implies that SNPs that are distant from CpG sites can also influence their methylation (Gibbs et al., 2010). In general, in these studies, multiple SNPs (most of them in high LD) do correlate with methylation levels of a single CpG site. Considering this and in order to minimize redundancy, we did not inspect all the SNP variants reported to influence methylation at every CpG site. Instead, we selected the SNP showing the best correlation with methylation for each site. Therefore, there are other SNPs displaying ASM for the same CpG sites in high LD with the reported ones that could be the ones involved in the effect on DNA methylation (Supplementary Table 7). Importantly, the two variants identified in our study highlight $\mathrm{CpG}$ sites that could have a role in drug dependence.

The rs6020251 variant was associated with drug dependence and also specifically with cocaine dependence in two independent samples and correlates with the methylation levels of cg17974185, that lies in the first intron of the CTNNBL1 gene (Fig. 3A). Noteworthy, this variant is in a region with multiple histone marks related to enhancer and promoter regions in multiple brain areas and is predicted to affect the binding of four TFBS (Table 2 and Supplementary Tables 5 and 6), reinforcing its role in regulation. Furthermore, this SNP has been reported to be an MQTL (methylation quantitative trait loci) in human fetal brain (Hannon et al., 2015). The CTNNBL1 is expressed in most tissues, including brain, and encodes a component of the PRP19-CDC5L protein complex, an integral part of the spliceosome involved in the activation of pre-mRNA splicing (van Maldegem et al., 2015). Interestingly, CTNNBL1 has been associated with obesity (Liu et al., 2008) and with memory (Papassotiropoulos et al., 2013), both related to drug dependence (Goodman and Packard, 2016; Kutlu and Gould, 2016; Volkow et al., 2013, Volkov et al., 2017). The effect of food in obesity resembles that of the drug in addiction, as both result in powerful 
Table 2

Functional annotations for the risk alleles of the significant associations with drug and cocaine dependence.

\begin{tabular}{|c|c|c|c|c|c|c|c|c|c|c|c|c|c|c|c|c|c|c|}
\hline \multirow[b]{2}{*}{ SNP } & \multirow[b]{2}{*}{$\begin{array}{l}\text { Risk } \\
\text { allele } \\
\end{array}$} & \multirow[b]{2}{*}{${ }^{\mathrm{a}}$ Gene } & \multicolumn{5}{|c|}{${ }^{\mathrm{b} E \text { Effect on methylation }}$} & \multicolumn{2}{|c|}{${ }^{\mathrm{c}}$ Epigenetic marks } & \multicolumn{5}{|c|}{${ }^{\mathrm{d}}$ Effect on expression } & \multicolumn{4}{|c|}{${ }^{\mathrm{e}}$ Effect on TFBS motifs } \\
\hline & & & Effect & CpG site & Tissue & $\begin{array}{l}\mathrm{p}- \\
\text { value }\end{array}$ & $\mathrm{R}^{2}$ & Enhancer & Promoter & Effect & Gene & Tissue & $\begin{array}{l}\text { Effect } \\
\text { size }\end{array}$ & $\begin{array}{l}\mathrm{p}- \\
\text { value }\end{array}$ & Effect & $\mathrm{TF}$ & $\begin{array}{l}\text { TFBS } \\
\text { score }\end{array}$ & $\begin{array}{l}\text { p- } \\
\text { value }\end{array}$ \\
\hline \multirow[t]{9}{*}{ rs11585570 } & C & $\begin{array}{l}E C H D C 2 \\
S C P 2\end{array}$ & $\uparrow$ & cg27535305 & $\begin{array}{l}\text { Frontal } \\
\text { Cortex }\end{array}$ & $\begin{array}{l}2.08 \mathrm{E}- \\
26\end{array}$ & 0.58 & - & - & $\downarrow$ & $E C H D C 2$ & $\begin{array}{l}\text { Cerebellar } \\
\text { Hemisphere }\end{array}$ & -0.26 & $\begin{array}{l}3.30 \mathrm{E}- \\
05\end{array}$ & $\uparrow$ & HOXA5 & 10.04 & $\begin{array}{l}1.69 \mathrm{E}- \\
04\end{array}$ \\
\hline & & & & & Pons & $\begin{array}{l}1.41 \mathrm{E}- \\
20\end{array}$ & 0.51 & & & & & Cerebellum & -0.31 & $\begin{array}{l}6.50 \mathrm{E}- \\
06\end{array}$ & & & & \\
\hline & & & & & $\begin{array}{l}\text { Temporal } \\
\text { Cortex }\end{array}$ & $\begin{array}{l}6.04 \mathrm{E}- \\
33\end{array}$ & 0.68 & & & & & $\begin{array}{l}\text { Nucleus } \\
\text { accumbens }\end{array}$ & -0.16 & $\begin{array}{l}3.00 \mathrm{E}- \\
06\end{array}$ & & & & \\
\hline & & & & & & & & & & & & Whole blood & -0.24 & $\begin{array}{l}6.60 \mathrm{E}- \\
36 \\
\end{array}$ & & & & \\
\hline & & & $\uparrow$ & $\operatorname{cg} 13461509^{*}$ & Cerebellum & $\begin{array}{l}1.91 \mathrm{E}- \\
10\end{array}$ & 0.32 & & & $\downarrow$ & $S C P 2$ & $\begin{array}{l}\text { Cerebellar } \\
\text { Hemisphere }\end{array}$ & -0.23 & $\begin{array}{l}9.90 \mathrm{E}- \\
07\end{array}$ & & & & \\
\hline & & & & & $\begin{array}{l}\text { Frontal } \\
\text { Cortex }\end{array}$ & $\begin{array}{l}2.10 \mathrm{E}- \\
10\end{array}$ & 0.27 & & & & & Cerebellum & -0.20 & $\begin{array}{l}6.10 \mathrm{E}- \\
05\end{array}$ & & & & \\
\hline & & & & & Temporal & $2.97 \mathrm{E}-$ & 0.30 & & & & & Cortex & -0.13 & $3.10 \mathrm{E}-$ & & & & \\
\hline & & & & & Cortex & 11 & & & & & & & & 05 & & & & \\
\hline & & & & & & & & & & & & Whole blood & -0.21 & $\begin{array}{l}8.10 \mathrm{E}- \\
21\end{array}$ & & & & \\
\hline \multirow[t]{4}{*}{ rs6020251 } & A & CTNNBL1 & $\uparrow$ & cg17974185 & Cerebellum & $\begin{array}{l}1.29 \mathrm{E}- \\
24\end{array}$ & 0.63 & 15 & 16 & - & - & - & - & - & $\uparrow$ & NKX3.2 & 9.02 & $\begin{array}{l}3.08 \mathrm{E}- \\
04\end{array}$ \\
\hline & & & & & $\begin{array}{l}\text { Frontal } \\
\text { Cortex }\end{array}$ & $\begin{array}{l}6.22 \mathrm{E}- \\
29\end{array}$ & 0.62 & & & & & & & & & NKX3.1 & 9.05 & $\begin{array}{l}2.88 \mathrm{E}- \\
04\end{array}$ \\
\hline & & & & & Pons & $\begin{array}{l}2.55 \mathrm{E}- \\
28\end{array}$ & 0.63 & & & & & & & & & ZNF354C & 8.08 & $\begin{array}{l}1.22 \mathrm{E}- \\
03\end{array}$ \\
\hline & & & & & $\begin{array}{l}\text { Temporal } \\
\text { Cortex }\end{array}$ & $\begin{array}{l}2.85 \mathrm{E}- \\
27\end{array}$ & 0.61 & & & & & & & & & ISL2 & 8.20 & $\begin{array}{l}5.08 \mathrm{E}- \\
04\end{array}$ \\
\hline
\end{tabular}

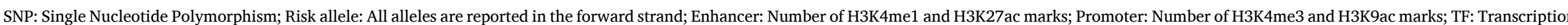

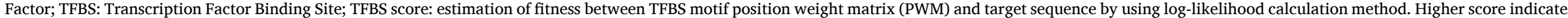

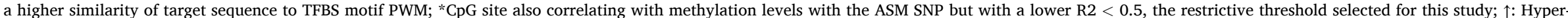
methylation/Higher similarity of target sequence to TFBS motif; $\downarrow$ : Hypomethylation/Underexpression; "-": No significant data for the SNP.

${ }^{\text {a }}$ Gene: Genes with the reported $\mathrm{CpG}$ sites lying in their possible regulatory regions (promotor or first intron).

b Described in Zhang et al. (2010) and Gibbs et al. (2010).

${ }^{\mathrm{c}}$ Histone marks found in brain areas.

d eQTL information for brain tissues and whole blood obtained from GTEx database.

e Information obtained from FeatSNP tool. 
reinforcing effects characterized by an increase of dopamine in brain (Volkow et al., 2013, Volkov et al., 2017). Additionally, it has been suggested that individuals presenting obesity or drug dependence have impairments in the dopaminergic pathways that mediate reward (Volkow et al., 2013, Volkov et al., 2017). About memory, it is known that drug use is related to maladaptive memories that support drug addiction and relapse. Moreover, drugs of abuse are often related to disrupted learning (Goodman and Packard, 2016; Kutlu and Gould, 2016).

The rs11585570 variant was found associated with drug and with cocaine dependence in the discovery sample but not in the replication dataset, although the pooled analysis yielded significant results. This variant correlates with differential levels of methylation of two CpG sites (cg27535305 and $\operatorname{cg} 13461509)$ that lie in the promoter regions of the SCP2 and ECHDC2 genes (Fig. 3B) and is also predicted to affect the binding of a transcription factor, HOXA5 (Table 2 and Supplementary Table 6). In general, DNA methylation in promoter regions inversely correlates with levels of gene expression (Li and Zhang, 2014), and this is observed with variant rs11585570, where the risk allele C is associated with hypermethylation of cg27535305 and cg13461509 in brain and to downregulation of SCP2 and ECHDC2 in brain and blood (Fig. 3B and Table 2). Furthermore, cg13461509 methylation also correlates with the expression of SCP2 in peripheral blood (Wu et al., 2018), pointing it out as a possible biomarker for the disorder. Remarkably, the variant rs11585570 is an eQTL for the ECHDC2 gene in the nucleus accumbens, a key area in addiction. Addictive drugs increase the extracellular concentration of dopamine in the nucleus accumbens, a neurotransmitter involved in reward and motivation, two main features of addiction (Di Chiara, 2002; Di Chiara et al., 2004; Koob and Volkow, 2016). This effect on gene expression in the nucleus accumbens is highlighting a possible window to be studied in depth to understand the specific role of this variant to drug dependence susceptibility. The SCP2 and ECHDC2 genes are expressed in most tissues, including brain. $E C H D C 2$ is involved in the metabolism of fatty acids and lipids and SCP2 plays a role in the lipid transfer between membranes and interestingly, was found increased in the brain of mice exposed to alcohol (Myers-Payne et al., 1996).

All these evidences and predictions together with the results obtained in the present work support the functional role of these variants at an epigenetic level, involving methylation of the CpG sites cg17974185, cg27535305 and cg13461509 in addiction. Furthermore, two of the highlighted genes (SCP2 and CTNNBL1) were previously linked to other disorders or conditions related to drug dependence (Liu et al., 2008; Myers-Payne et al., 1996; Papassotiropoulos et al., 2013).

There are several strengths and limitations of this study that should be discussed. Multiple factors may influence the statistical power of an association study, for instance sample size, homogeneity of the sample used or prevalence of the disease in the general population (Nsengimana and Bishop, 2017). Sample size is one of the most important elements that contribute to statistical power and it directly correlates with it (Hong and Park, 2012). The sample size of this study is substantially larger than the ones used in previous studies on drug dependence; nonetheless, it is still limited taking into account that most of the variants contributing to complex disorders like addiction have modest effects. Homogeneity of the sample used is also relevant, since heterogeneity could lead to false positives (Nsengimana and Bishop, 2017). In order to control this, all subjects included in our study were Spanish, with European ancestry, recruited in Barcelona (Spain), sex ratios were similar in cases and controls, and all patients were evaluated in the same hospital and under the same clinical assessment. In the present study, we used non-screened controls from the general population, as previously done by several GWAS of drug addiction (Cabana-Domínguez et al., 2019; Ikeda et al., 2013; Johnson et al., 2015; Kalsi et al., 2016). Although we could not assess drug dependence in control individuals, the impact of the contamination of cases within the control sample is expected to be low, given that only $0.7 \%$ of the global population aged 15-64 suffer from illicit drug use disorders (UNODC,
2019) and $5.1 \%$ of the global population aged 15 years and older suffer from alcohol use disorders (World Health Organization, 2018). However, as this design could eventually dilute positive findings (false negative results), other studies in worldwide cohorts are needed to support the results obtained here and to further delineate the contribution of ASM to the disorder. In association studies it is also necessary to minimize marker redundancies to improve statistical power. Lastly, replication studies in independent samples are widely used to confirm the association and direction of the effect of genetic variants identified in a discovery sample (Chanock et al., 2007). When a variant replicates in a follow-up sample, its association with the evaluated phenotype is reinforced (Chanock et al., 2007). In this work, although the associations found in the discovery sample were nominal, we were able to replicate one of them in a second sample that doubled the size of the discovery. Moreover, this hit and a second one showed association in a pooled analysis combining the discovery and replication samples, strengthening the results obtained.

In conclusion, we report an association of rs6020251 and rs11585570 with drug dependence and with cocaine dependence. Interestingly, these SNPs correlate with methylation levels of nearby CpG sites (Gibbs et al., 2010; Zhang et al., 2010) that are located in possible regulatory regions of the CTNNBL1 or the SCP2 and ECHDC2 genes, respectively. In addition, the risk allele of rs11585570 is associated with a lower expression of SCP2 and ECHDC2 in brain and blood. Our results highlight these genes as new candidates for involvement in the susceptibility to drug dependence, although further functional studies are needed to ascertain their possible role in the disorder.

\section{Role of funding source}

This work was supported by major financial support received by BC from the Spanish 'Ministerio de Ciencia e Innovación' [grant numbers SAF2015-68341-R and RTI2018-100968-B-I00] and 'Agència de Gestió d'Ajuts Universitaris i de Recerca - AGAUR, Generalitat de Catalunya' [grant number 2017-SGR-738]. MR received funding from the 'Instituto de Salud Carlos III' [grant numbers PI16/01505, PI17/00289 and PI18/ 01788] and was cofinanced by the European Regional Development Fund - ERDF, from the 'Pla estratègic de recerca i innovació en salut PERIS, Generalitat de Catalunya' [grant number SLT006/17/287] and the 'Agència de Gestió d'Ajuts Universitaris i de Recerca - AGAUR, Generalitat de Catalunya' [grant number 2017SGR1461]. BC and MR also received funding from the European Union H2020 Program [grant numbers H2020/2014-2020, n667302 (CoCA) and n ${ }^{\circ} 728018$ (Eat2beNICE)]. LP-C was supported by 'Ministerio de Educación, Cultura y Deporte' [grant number FPU15/03867] and by 'Generalitat de Catalunya' [grant number 2016 FI_B 00728], JC-D by the H2020 CoCA and Eat2beNICE projects and NF-C by a contract of the 'Centro de Investigación Biomédica en Red de Enfermedades Raras - CIBERER'. CS-M was a recipient of a Sara Borrell contract from the 'Instituto de Salud Carlos III' [grant number CD15/00199] and MR of a Miguel de Servet contract from the 'Instituto de Salud Carlos III' [grant numbers CP09/ 00119 and CPII15/00023]. The work was also supported by the ECNP Network 'ADHD across the Lifespan' (https://www.ecnp.eu/researchinnovation/ECNP-networks/List-ECNP-Networks/).

\section{CRediT authorship contribution statement}

Laura Pineda-Cirera: Methodology, Formal analysis, Investigation, Writing - original draft, Writing - review \& editing, Visualization. Judit Cabana-Domínguez: Investigation, Visualization, Writing - review \& editing. Lara Grau-López: Data curation, Resources. Constanza Daigre: Resources. Cristina Sánchez-Mora: Data curation, Resources. Raul Felipe Palma-Álvarez: Resources. Josep Antoni Ramos-Quiroga: Resources, Writing - review \& editing, Visualization. Marta Ribasés: Resources, Writing - review \& editing, Funding acquisition. Bru Cormand: Conceptualization, Methodology, Writing - original draft, 
Writing - review \& editing, Visualization, Supervision, Project administration, Funding acquisition. Noèlia Fernàndez-Castillo: Conceptualization, Methodology, Investigation, Writing - original draft, Writing review \& editing, Visualization, Supervision.

\section{Declaration of competing interest}

The authors declare the following financial interests/personal relationships which may be considered as potential competing interests. JAR-Q was on the speakers' bureau and/or acted as consultant for EliLilly, Janssen-Cilag, Novartis, Shire, Takeda, Bial, Shionogui, Lundbeck, Almirall, Braingaze, Sincrolab, Medice and Rubió in the last 5 years. He also received travel awards (air tickets + hotel) for taking part in psychiatric meetings from Janssen-Cilag, Rubió, Shire, Takeda, Shionogui, Bial, Medice and Eli- Lilly. The Department of Psychiatry chaired by him received unrestricted educational and research support from the following companies in the last 5 years: Eli-Lilly, Lundbeck, Janssen- Cilag, Actelion, Shire, Ferrer, Oryzon, Roche, Psious, and Rubió. RFP-A has received fees to give talks for Exeltis, Lundbeck, and Takeda. LG-L has received fees to give talks for Janssen and Lundbeck. The other authors declare no conflict of interest.

\section{Acknowledgements}

The authors thank technical and human support provided by SGIker of UPV/EHU. The authors are also grateful to "Agència de Salut Pública de Barcelona", the Blood and Tissues Bank of Barcelona donors, patients, families and clinical collaborators who contributed to the recruitment of patients (Dra. Nieves Martinez-Luna, Dra. Elena Ros-Cucurull, Eduardo Castrillo and Miguel Ángel Cantillo).

\section{Appendix A. Supplementary data}

Supplementary data to this article can be found online at https://doi. org/10.1016/j.jpsychires.2020.07.044.

\section{References}

Ajonijebu, D.C., Abboussi, O., Russell, V.A., Mabandla, M.V., Daniels, W.M.U., 2017. Epigenetics: a link between addiction and social environment. Cell. Mol. Life Sci. 74, 2735-2747.

Andrews, S.V., Ellis, S.E., Bakulski, K.M., Sheppard, B., Croen, L.A., Hertz-Picciotto, I., Newschaffer, C.J., Feinberg, A.P., Arking, D.E., Ladd-Acosta, C., Fallin, M.D., 2017 Cross-tissue integration of genetic and epigenetic data offers insight into autism spectrum disorder. Nat. Commun. 8, 1011.

Barrett, J.C., 2009. Haploview: visualization and analysis of SNP genotype data. Cold Spring Harb. Protoc, 4, 1-5.

Bashtrykov, P., Jeltsch, A., 2017. Epigenome editing in the brain. In: Delgado-Morales, R (Ed.), Neuroepigenomics in Aging and Disease. Advances in Experimental Medicine and Biology. Springer, Cham, pp. 409-424. https://doi.org/10.1007/978-3-31953889-1.

Brown, A.N., Feng, J., 2017. Drug addiction and DNA modifications. In: DelgadoMorales, R. (Ed.), Neuroepigenomics in Aging and Disease. Advances in Experimental Medicine and Biology. Springer, Cham, pp. 105-125.

Bühler, K.-M., Giné, E., Echeverry-Alzate, V., Calleja-Conde, J., de Fonseca, F.R., López Moreno, J.A., 2015. Common single nucleotide variants underlying drug addiction: more than a decade of research. Addiction Biol. 20, 845-871.

Cabana-Domínguez, J., Shivalikanjli, A., Fernàndez-Castillo, N., Cormand, B., 2019. Genome-wide association meta-analysis of cocaine dependence: shared genetics with comorbid conditions. Prog. Neuro-Psychopharmacol. Biol. Psychiatry 94, 109667. https://doi.org/10.1016/j.pnpbp.2019.109667.

Cadet, J.L., McCoy, M.T., Jayanthi, S., 2016. Epigenetics and addiction. Clin. Pharmacol Ther. 99, 502-511.

Chanock, S.J., Manolio, T., Boehnke, M., Boerwinkle, E., Hunter, D.J., Thomas, G., Hirschhorn, J.N., Abecasis, G., Altshuler, D., Bailey-Wilson, J.E., Brooks, L.D., Cardon, L.R., Daly, M., Donnelly, P., Fraumeni, J.F., Freimer, N.B., Gerhard, D.S., Gunter, C., Guttmacher, A.E., Guyer, M.S., Harris, E.L., Hoh, J., Hoover, R., Kong, C A., Merikangas, K.R., Morton, C.C., Palmer, L.J., Phimister, E.G., Rice, J.P., Roberts, J., Rotimi, C., Tucker, M.A., Vogan, K.J., Wacholder, S., Wijsman, E.M., Winn, D.M., Collins, F.S., 2007. Replicating genotype-phenotype associations. Nature 447, 655-660. https://doi.org/10.1038/447655a.

Chuang, L.-C., Kao, C.-F., Shih, W.-L., Kuo, P.-H., 2013. Pathway analysis using information from allele-specific gene methylation in genome-wide association studies for bipolar disorder. PloS One 8, e53092.
Di Chiara, G., 2002. Nucleus accumbens shell and core dopamine: differential role in behavior and addiction. Behav. Brain Res. 137, 75-114.

Di Chiara, G., Bassareo, V., Fenu, S., De Luca, M.A., Spina, L., Cadoni, C., Acquas, E., Carboni, E., Valentini, V., Lecca, D., 2004. Dopamine and drug addiction: the nucleus accumbens shell connection. Neuropharmacology 47, 227-241.

Do, C., Lang, C.F., Lin, J., Darbary, H., Krupska, I., Gaba, A., Petukhova, L., Vonsattel, J. P., Gallagher, M.P., Goland, R.S., Clynes, R.A., Dwork, A., Kral, J.G., Monk, C., Christiano, A.M., Tycko, B., 2016. Mechanisms and disease associations of haplotype-dependent allele-specific DNA methylation. Am. J. Hum. Genet. 98, 934-955. https://doi.org/10.1016/j.ajhg.2016.03.027.

Do, C., Shearer, A., Suzuki, M., Terry, M.B., Gelernter, J., Greally, J.M., Tycko, B., 2017. Genetic-epigenetic interactions in cis: a major focus in the post-GWAS era. Genome Biol. 18, 120.

Ducci, F., Goldman, D., 2012. The genetic basis of addictive disorders. Psychiatr. Clin. 35, 495-519.

Edenberg, H.J., Gelernter, J., Agrawal, A., 2019. Genetics of. Alcoholism. Curr. Psychiatry Rep. 21, 26. https://doi.org/10.1007/s11920-019-1008-1.

Fernàndez-Castillo, N., Roncero, C., Grau-Lopez, L., Barral, C., Prat, G., RodriguezCintas, L., Sánchez-Mora, C., Gratacòs, M., Ramos-Quiroga, J.A., Casas, M., Ribasés, M., Cormand, B., 2013. Association study of 37 genes related to serotonin and dopamine neurotransmission and neurotrophic factors in cocaine dependence. Gene Brain Behav. 12, 39-46.

First, M.B., Spitzer, S.R., Gibbon, M., Williams, J.B.M., 1997. Structured Clinical Interview for DSM-IV Axis I Disorders (SCID). Am. Psychiatr. Press.

Gagliano, S.A., Ptak, C., Mak, D.Y.F., Shamsi, M., Oh, G., Knight, J., Boutros, P.C., Petronis, A., 2016. Allele-skewed DNA modification in the brain: relevance to a schizophrenia GWAS. Am. J. Hum. Genet. 98, 956-962.

Gaur, U., Li, K., Mei, S., Liu, G., 2013. Research progress in allele-specific expression and its regulatory mechanisms. J. Appl. Genet. 54, 271-283.

Gibbs, J.R., van der Brug, M.P., Hernandez, D.G., Traynor, B.J., Nalls, M.A., Lai, S.L., Arepalli, S., Dillman, A., Rafferty, I.P., Troncoso, J., Johnson, R., Zielke, H.R., Ferrucci, L., Longo, D.L., Cookson, M.R., Singleton, A.B., 2010. Abundant quantitative trait loci exist for DNA methylation and gene expression in Human Brain. PLoS Genet. 6, e1000952.

González, J.R., Armengol, L., Solé, X., Guinó, E., Mercader, J.M., Estivill, X., Moreno, V., 2007. SNPassoc: an R package to perform whole genome association studies. Bioinformatics 23, 644-645.

Goodman, J., Packard, M.G., 2016. Memory systems and the addicted brain. Front. Psychiatr. 7, 24.

Hamilton, P.J., Nestler, E.J., 2019. Epigenetics and addiction. Curr. Opin. Neurobiol. 59, 128-136. https://doi.org/10.1016/j.conb.2019.05.005.

Hannon, E., Spiers, H., Viana, J., Pidsley, R., Burrage, J., Murphy, T.M., Troakes, C., Turecki, G., O'Donovan, M.C., Schalkwyk, L.C., Bray, N.J., Mill, J., 2015. Methylation QTLs in the developing brain and their enrichment in schizophrenia risk loci. Nat. Neurosci. 19, 48-54.

Hong, E.P., Park, J.W., 2012. Sample size and statistical power calculation in genetic association studies. Genomics Inform 10, 117. https://doi.org/10.5808/ gi.2012.10.2.117.

Ikeda, M., Okahisa, Y., Aleksic, B., Won, M., Kondo, N., Naruse, N., Aoyama-Uehara, K., Sora, I., Iyo, M., Hashimoto, R., Kawamura, Y., Nishida, N., Miyagawa, T., Takeda, M., Sasaki, T., Tokunaga, K., Ozaki, N., Ujike, H., Iwata, N., 2013. Evidence for shared genetic risk between methamphetamine-induced psychosis and schizophrenia. Neuropsychopharmacology 38, 1864-1870. https://doi.org/ 10.1038/npp.2013.94.

Jensen, K.P., 2016. A review of genome-wide association studies of stimulant and opioid use disorders. Mol. neuropsychiatry 2, 37-45.

Johnson, E.O., Hancock, D.B., Levy, J.L., Gaddis, N.C., Page, G.P., Glasheen, C. Saccone, N.L., Bierut, L.J., Kral, A.H., 2015. KAT2B polymorphism identified for drug abuse in African Americans with regulatory links to drug abuse pathways in human prefrontal cortex. Addiction Biol. 21, 1217-1232.

Kalsi, G., Euesden, J., Coleman, J.R.I., Ducci, F., Aliev, F., Newhouse, S.J., Liu, X., Ma, X., Wang, Y., Collier, D.A., Asherson, P., Li, T., Breen, G., 2016. Genome-wide association of heroin dependence in han Chinese. PloS One 11, e0167388. https:// doi.org/10.1371/journal.pone.0167388.

Klose, R.J., Bird, A.P., 2006. Genomic DNA methylation: the mark and its mediators. Trends Biochem. Sci. 31, 89-97. https://doi.org/10.1016/j.tibs.2005.12.008.

Koob, G.F., Volkow, N.D., 2016. Neurobiology of addiction: a neurocircuitry analysis. The Lancet Psychiatry 3, 760-773. https://doi.org/10.1016/S2215-0366(16)00104-

Kutlu, M.G., Gould, T.J., 2016. Effects of drugs of abuse on hippocampal plasticity and hippocampus-dependent learning and memory: contributions to development and maintenance of addiction. Learn. Mem. 23, 515-533.

Ladd-Acosta, C., Pevsner, J., Sabunciyan, S., Yolken, R.H., Webster, M.J., Dinkins, T., Callinan, P.A., Fan, J.-B., Potash, J.B., Feinberg, A.P., 2007. DNA methylation signatures within the human brain. Am. J. Hum. Genet. 81, 1304-1315.

Li, E., Zhang, Y., 2014. DNA methylation in mammals. Cold Spring Harb. Perspect. Biol. 6, a019133. https://doi.org/10.1101/cshperspect.a019133.

Liu, Y.J., Liu, X.G., Wang, L., Dina, C., Yan, H., Liu, J.F., Levy, S., Papasian, C.J., Drees, B. M., Hamilton, J.J., Meyre, D., Delplanque, J., Pei, Y.F., Zhang, L., Recker, R.R. Froguel, P., Deng, H.W., 2008. Genome-wide association scans identified CTNNBL1 as a novel gene for obesity. Hum. Mol. Genet. 17, 1803-1813. https://doi.org/ 10.1093/hmg/ddn072.

Lonsdale, J., Thomas, J., Salvatore, M., Phillips, R., Lo, E., Shad, S., Hasz, R., Walters, G., Garcia, F., Young, N., Foster, B., Moser, M., Karasik, E., Gillard, B., Ramsey, K., Sullivan, S., Bridge, J., Magazine, H., Syron, J., Fleming, J., Siminoff, L., Traino, H., Mosavel, M., Barker, L., Jewell, S., Rohrer, D., Maxim, D., Filkins, D., Harbach, P., 
Cortadillo, E., Berghuis, B., Turner, L., Hudson, E., Feenstra, K., Sobin, L., Robb, J. Branton, P., Korzeniewski, G., Shive, C., Tabor, D., Qi, L., Groch, K., Nampally, S. Buia, S., Zimmerman, A., Smith, A., Burges, R., Robinson, K., Valentino, K., Bradbury, D., Cosentino, M., Diaz-Mayoral, N., Kennedy, M., Engel, T., Williams, P., Erickson, K., Ardlie, K., Winckler, W., Getz, G., DeLuca, D., MacArthur, Daniel, Kellis, M., Thomson, A., Young, T., Gelfand, E., Donovan, M., Meng, Y., Grant, G., Mash, D., Marcus, Y., Basile, M., Liu, J., Zhu, J., Tu, Z., Cox, N.J., Nicolae, D.L., Gamazon, E.R., Im, H.K., Konkashbaev, A., Pritchard, J., Stevens, M., Flutre, T., Wen, X., Dermitzakis, E.T., Lappalainen, T., Guigo, R., Monlong, J., Sammeth, M. Koller, D., Battle, A., Mostafavi, S., McCarthy, M., Rivas, M., Maller, J., Rusyn, I., Nobel, A., Wright, F., Shabalin, A., Feolo, M., Sharopova, N., Sturcke, A., Paschal, J. Anderson, J.M., Wilder, E.L., Derr, L.K., Green, E.D., Struewing, J.P., Temple, G. Volpi, S., Boyer, J.T., Thomson, E.J., Guyer, M.S., Ng, C., Abdallah, A., Colantuoni, D., Insel, T.R., Koester, S.E., Roger Little, A., Bender, P.K., Lehner, T., Yao, Y., Compton, C.C., Vaught, J.B., Sawyer, S., Lockhart, N.C., Demchok, J., Moore, H.F., 2013. The genotype-tissue expression (GTEx) project. Nat. Genet. 45 , 580-585. https://doi.org/10.1038/ng.2653.

Ma, C., Madden, P., Gontarz, P., Wang, T., Zhang, B., 2019. FeatSNP : an interactive database for brain-specific epigenetic annotation of human SNPs. Front. Genet. 10, 262.

Meaburn, E.L., Schalkwyk, L.C., Mill, J., 2010. Allele-specific methylation in the human genome: implications for genetic studies of complex disease. Epigenetics 5, 578-582.

Miller, S.A., Dykes, D.D., Polesky, H.F., 1988. A simple salting out procedure for extracting DNA from human nucleated cells. Nucleic Acids Res. 16, 1215.

Mulligan, M.K., 2019. Genetic factors in cannabinoid use and dependence. In: Bukiya, A. (Ed.), Recent Advances in Cannabinoid Physiology and Pathology. Advances in Experimental Medicine and Biology. Springer, Cham, pp. 129-150. https://doi.org/ 10.1007/978-3-030-21737-2_7.

Myers-Payne, S.C., Fontaine, R.N., Loeffler, A., Pu, L., Rao, A.M., Kier, A.B., Wood, W.G., Schroeder, F., 1996. Effects of chronic ethanol consumption on sterol transfer proteins in mouse brain. J. Neurochem. 66, 313-320.

Nsengimana, J., Bishop, D.T., 2017. Design considerations for genetic linkage and association studies. In: Elston, R. (Ed.), Statistical Human Genetics. Methods in Molecular Biology. Humana Press, New York, NY, pp. 257-281. https://doi.org/ 10.1007/978-1-4939-7274-6_13.

Papassotiropoulos, A., Stefanova, E., Vogler, C., Gschwind, L., Ackermann, S., Spalek, K., Rasch, B., Heck, A., Aerni, A., Hanser, E., Demougin, P., Huynh, K.D., Luechinger, R.,
Klarhöfer, M., Novakovic, I., Kostic, V., Boesiger, P., Scheffler, K., De Quervain, D.J. F., 2013. A genome-wide survey and functional brain imaging study identify CTNNBL1 as a memory-related gene. Mol. Psychiatr. 18, 255-263.

Pineda-Cirera, L., Shivalikanjli, A., Cabana-Domínguez, J., Demontis, D., Rajagopal, V. M., Børglum, A.D., Faraone, S.V., Cormand, B., Fernàndez-Castillo, N., 2019. Exploring genetic variation that influences brain methylation in attention-deficit/ hyperactivity disorder. Transl. Psychiatry 9, 242. https://doi.org/10.1038/s41398019-0574-7.

Tycko, B., 2010. Allele-specific DNA methylation: beyond imprinting. Hum. Mol. Genet. 19, R210-R220. https://doi.org/10.1093/hmg/ddq376.

UNODC, 2019. World Drug Report Sales No. E.19.XI.9.

van Maldegem, F., Maslen, S., Johnson, C.M., Chandra, A., Ganesh, K., Skehel, M., Rada, C., 2015. CTNNBL1 facilitates the association of CWC15 with CDC5L and is required to maintain the abundance of the Prp19 spliceosomal complex. Nucleic Acids Res. 43, 7058-7069.

Volkov, N.D., Wise, R.A., Baler, R., 2017. The dopamine motive system: implications for drug and food addiction. Nature Reviews Neuroscience 18, 741-752. https://doi. org/10.1038/nrn.2017.130.

Volkow, N.D., Wang, G.J., Tomasi, D., Baler, R.D., 2013. Obesity and addiction: neurobiological overlaps. Obes. Rev. 14, 2-18. https://doi.org/10.1111/j.1467. 789X.2012.01031.x.

Ward, L.D., Kellis, M., 2016. HaploReg v4: systematic mining of putative causal variants, cell types, regulators and target genes for human complex traits and disease. Nucleic Acids Res. 44, D877-D881.

World Health Organization, 2018. Global Status Report on Alcohol and Health. Licence: BY-NC-SA 3.0 IGO, Geneva.

Wu, C., Pan, W., 2019. Integration of methylation QTL and enhancer-target gene maps with schizophrenia GWAS summary results identifies novel genes. Bioinformatics 35, 3576-3583.

Wu, Y., Zeng, J., Zhang, F., Zhu, Z., Qi, T., Zheng, Z., Lloyd-Jones, L.R., Marioni, R.E. Martin, N.G., Montgomery, G.W., Deary, I.J., Wray, N.R., Visscher, P.M., McRae, A. F., Yang, J., 2018. Integrative analysis of omics summary data reveals putative mechanisms underlying complex traits. Nat. Commun. 9, 918.

Zhang, D., Cheng, L., Badner, J.a., Chen, C., Chen, Q., Luo, W., Craig, D.W., Redman, M., Gershon, E.S., Liu, C., 2010. Genetic control of individual differences in gene-specific methylation in human brain. Am. J. Hum. Genet. 86, 411-419. 